\title{
Demand Response Baseline Load Forecasting Based on the Combination of Time Series and Kalman Filter
}

\author{
Jun Dong, Shilin Nie* \\ School of Economics and Management, North China Electric Power University, Beijing, China \\ Email address: \\ dongjun624@126.com (Jun Dong),ns103ncepu@163.com (Shilin Nie) \\ ${ }^{*}$ Corresponding author
}

To cite this article:

Jun Dong, Shilin Nie. Demand Response Baseline Load Forecasting Based on the Combination of Time Series and Kalman Filter. American Journal of Electrical Power and Energy Systems. Vol. 8, No. 3, 2019, pp. 71-76. doi: 10.11648/j.epes.20190803.11

Received: May 16, 2019; Accepted: June 13, 2019; Published: June 26, 2019

\begin{abstract}
The customer baseline load is an important reference for the industrial and commercial users to participate in the demand response project, and is affected by various factors such as the environment and user electricity usage. In order to improve the accuracy of the baseline load forecasting of industrial and commercial users, a demand response baseline load forecasting model based on time series and Kalman filter combination is proposed. The marginal contribution rate of the single forecasting model to the combined model is obtained by the Shapley value method, then gets optimal prediction results. The case results show that the Kalman filter model has higher prediction accuracy in the period of stable load fluctuation, and the ARMA model has higher prediction accuracy in the period of large load fluctuation, and the combined prediction model combines the advantages of both models and reduces the single model is affected by the time factor in the prediction process, which improves the overall prediction accuracy and expands the scope of application.
\end{abstract}

Keywords: Baseline Load, Demand Response, Load Forecasting, ARMA, Kalman Filter

\section{Introduction}

Demand response (DR) is widely recognized as a key technology to improve the flexibility of power system and the reliability of power supply, which is also a more economical and environmentally friendly method than traditional ones. [1] As an effective means of power demand side management, replacing supply-side energy with user-side resources using price signals and incentive mechanisms to guide users to optimize power usage is of great significance in mitigating grid pressure and maintaining safe operation of the grid. At present, the research on DR are mainly divided into price-based DR and incentive-based DR. In price-based DR, users change their power consumption according to the price of electricity, which means, when the power system approaches the peak value, the price of electricity rises, and the user automatically reduces the demand for electricity or shifts it to off-peak hours, which serves to cut the peak and fill the valley, helping the power system to run stably; In the incentive-based DR, the user needs to sign a response contract with the DR implementing agency, and the DR implementing agency will reward and punish users according to the user's contract performance. [2] However, to evaluate the effectiveness of various DR projects, a key step is to determine the baseline load of users participating in the DR project. The baseline load provides a data reference for quantitative evaluation of the degree of user load reduction. It is the basis for DR implementation agencies to implement rewards and punishments for customers, and is a measure to evaluate the effectiveness of DR projects.

The general definition of baseline load refers to the actual load of the user under the condition that the user does not perform the response during the demand response day. The user load reduction amount is calculated by using the baseline load and the actual load after the response is executed, [3-4] that is, the baseline load - the actual load $=$ the load reduction amount. At present, a large number of foreign research on the baseline load, and due to the relative lag in demand response in China, the current focus is on short-term load forecasting of power, [5-7] And the researches on the baseline load mainly take a page from overseas researches, summarize the results and improve the existing methods. [8-10] Foreign calculation methods of user baseline load are relatively rich, [11-13] New 
England ISO created a calculation method based on the user type, If the user has not previously participated in any requirements response items, the baseline load is calculated as an hourly average for the previous business day (except holidays and other event days). That is: Customer Baseline = (Sum Meter KW value for the hour)/5. If the user has previously been involved in a demand response project, the baseline load calculation formula is: new Baseline $=$ Previous day Baseline*0.9 + current day metered $\mathrm{kW}^{*} 0.1$. The new baseline load calculation formula avoids user speculation by assigning a weighted coefficient of the previous day and the same day; New York ISO established a baseline load calculation method for a recent demand response project, through selecting 5 days with the highest average daily load as reference data from historical data in the past ten days, and the prediction accuracy of the method is relatively low, but easy to operate, compared with other ISO, The California ISO calculates the 24-hour load on the event day by selecting the average of the three highest load days in the 10 days prior to the event date (excluding holidays and other event days); There is a similarity between PJM and New York ISO in baseline load calculation. They both use the load average of previous ten working days, but the selection of the first 5 Days of Load data method is different; The $+90^{\circ} \mathrm{F}$ Average Day predict the baseline load of the demand response day by selecting a historical load value that exceeds a certain temperature, whose disadvantage is that if there are inadequate amounts of data exceeding a certain temperature, prediction accuracy will decline.

Since the load of the power system exhibits a non-stationary fluctuation over a certain range with time, the above-mentioned baseline load calculation method is based on the average load value of the historical typical day, and is a smooth calculation method. Consequently, in order to improve the accuracy of baseline load forecasting of industrial and commercial users with non-stationary fluctuations, a demand response baseline load forecasting model based on time series and Kalman filter combination is proposed. The Kalman filter algorithm can solve the non-stationary stochastic process by using the recursive algorithm to establish the prediction equation and the update equation. By simulating the spatial state of the prediction system and performing feedback correction through the observations, the past and current states of the prediction system can be estimated effectively, and the accurate tracking of the dynamic system can be realized. At the same time, in the state of data loss, the time series contains various components that affect the system, accurately reflects the characteristics of the future development trend of the system, improves the weakness of Kalman filter tracking failure in the case of data loss, and obtains the optimal prediction result.

\section{Kalman Filter Model}

Kalman filter algorithm is proposed for the first time by Kalman in 1960, and the recursive method is used to solve the problem of linear filtering of data Kalman filter algorithm is an optimal estimation technique, [14] which can also estimate the past, present and future state of the target without the specific properties of the target model.

The state model and observation model formula of Kalman filter algorithm are as follows:

$$
\begin{gathered}
x_{k}=A x_{k-1}+w_{k-1} \\
z_{k}=H x_{k}+v_{k}
\end{gathered}
$$

Where the $x_{k}$ is the state value of the moment $k$, the $z_{k}$ is the observation value of the moment $k, A$ is the state transfer matrix, $H$ is the order of the Kalman gain matrix $m \times n, w_{k}$ and $v_{k}$ represents the target system process excitation noise and observation noise, And assume that they are independent of each other, normal distribution of white noise.

$$
\begin{aligned}
& p(w) \sim N(0, Q) \\
& p(v) \sim N(0, R)
\end{aligned}
$$

Where the covariance matrix $Q$ representing the process noise $w_{k}, R$ represents the covariance matrix of the observed noise $v_{k}$.

The Kalman filter algorithm uses the feedback control method to estimate the state of the variable, calculates the current state variable and the error covariance estimate forward through the time update process, and constructs the priori estimation of the next time state. In the state updating process, the prior estimation and the new measurement value are combined to construct the updated posterior estimation by feedback.

The time update equation is:

$$
\begin{aligned}
& \hat{x}_{k}^{-}=A \hat{x}_{k-1}+w_{k-1} \\
& P_{k}^{-}=A P_{k-1} A^{T}+Q
\end{aligned}
$$

Where $\hat{x}_{k}^{-}$is the priori estimate of the system moment $k, P_{k}^{-}$ is the covariance of the priori estimation error.

The status update equation is:

$$
\begin{gathered}
K_{k}=P_{k}^{-} H^{T}\left(H P_{k}^{-} H^{T}+R\right)^{-1} \\
\hat{x}_{k}=\hat{x}_{k}^{-}+K_{k}\left(z_{k}-H \hat{x}_{k}^{-}\right) \\
P_{k}=\left(I-K_{k} H\right) P_{k}^{-}
\end{gathered}
$$

Where the $K_{k}$ is Kalman gain, $I$ is the unit matrix. In the process of State update, the predicted value $\hat{x}_{k}^{-}$and $p_{k}^{-}$ are obtained by using the time update equation, and the estimated value $\hat{x}_{k}$ and error covariance $p_{k}$ at time $k$ are obtained by combining the observation value $z_{k}$ as the input variable. 


\section{ARMA Model}

ARMA model is a basic linear time series model, which has been widely used in power load forecasting and is relatively mature. In the process of prediction, the ARMA model takes into account both historical data and the influence of random interference of historical data on the prediction results. [15] Its predictive model can be expressed as $\operatorname{ARMA}(p, q)$, which is:

$$
\begin{gathered}
Y_{t}=\varphi_{1} X_{t-1}+\varphi_{2} X_{t-2}+\ldots+\varphi_{p} X_{t-p}- \\
\theta_{1} \varepsilon_{t-1}-\theta_{2} \varepsilon_{t-2}-\ldots-\theta_{q} \varepsilon_{t-q}+\varepsilon_{t}
\end{gathered}
$$

Where $\varphi_{i}(i=1,2,3 \ldots \ldots p)$ is the autoregressive coefficient, $\quad \theta_{i}(i=1,2,3 \ldots \ldots \mathrm{q})$ is the sliding average coefficient, $\varepsilon_{t}$ is the white noise sequence.

The ARMA Predictive modeling steps are as follows:

Step 1: Verify whether the historical load data is stable;

Step 2: If not smooth, the differential treatment is carried out, otherwise the order of the ARMA model is determined;
Step 3: Using EVIEWS Software, the estimated parameters of the model are obtained.

Step 4: Build the model and make prediction.

\section{Optimization Combination Prediction Model}

Kalman filtering is an optimal estimation technique. It uses the minimum mean square error as the criterion to perform mathematical recursive calculation. It uses the state at the previous moment of the system to obtain the optimal value at the next moment, which is suitable for the prediction of dynamic and discrete systems. The ARMA model considers the effects of multiple components on the system, and the trend of linear fitting systems is also applicable to the prediction of dynamic and discrete systems. Therefore, the two models have the characteristics of consistency and complementarity.

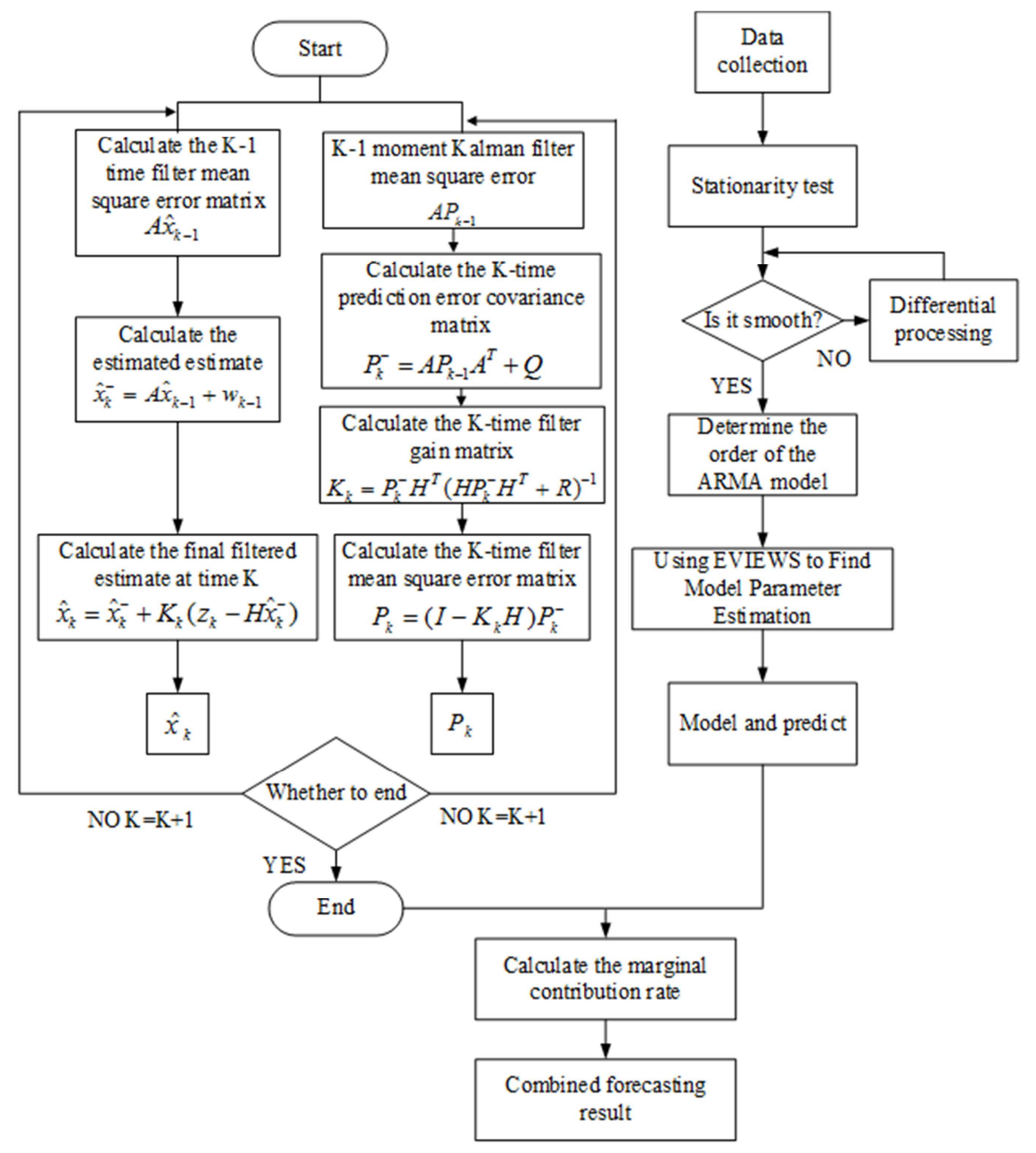

Figure 1. Flow chart of combined prediction model.

In view of the data loss caused by Kalman filtering's failure to track the system, the prediction results are biased greatly and the ARMA model cannot fully consider the influence of various factors on the system in the prediction process. In this paper, the Kalman filter is combined with the ARMA model by the Shapley value method. The optimal combination prediction result is obtained by calculating the marginal contribution rate of the single model prediction result to the 
combined model. The combined prediction model not only ensures that the combined model can achieve optimal prediction results, but also avoids the failure of data tracking missing Kalman filter target tracking, and brings time factors into the model. The calculation process of the combined forecasting model is: 1) calculating the Kalman filter prediction result; 2) calculating the ARMA model prediction result; 3) using the Shapley value method to calculate the marginal contribution rate of the single model to the combined prediction model, and obtaining the optimal prediction result. Figure 1 shows the combined forecasting model process.

The Shapley Value method is a kind of equitable distribution of benefits according to the marginal contribution of individuals to the system. Assuming that the Kalman filter model and ARMA model are two independent individuals of the system, the prediction results are $x_{1}, x_{2}$, and they do not affect each other, and the marginal contribution rate to the system is $K_{1}$ and $K_{2}$, the optimal result of the system is $X$. Since there are only two individuals in the system, a linear combination is used to solve. [16]

$$
\left\{\begin{array}{l}
X=K_{1} x_{1}+K_{2} x_{2} \\
K_{1}+K_{2}=1
\end{array}\right.
$$

Where $x_{1}$ and $x_{2}$ are the vector of the dimension $1 \times n$.

Let the absolute error between the predicted and actual values of the $\mathrm{i}$-th model is $e_{i t}$ :

$$
e_{i t}=x_{i t}-Y_{i t}
$$

The absolute error between the combined predicted value and the actual value is $e_{t}$ :

$$
\begin{aligned}
e_{t} & =X_{t}-Y_{t}=K_{1} x_{1}+K_{2} x_{2}-Y_{t} \\
& =\sum_{i}^{2} k_{i} e_{i t}
\end{aligned}
$$

The marginal contribution rate $K_{1}$ and $K_{2}$ are solved by finding absolute squared and minimum error:

$$
\begin{aligned}
f & =\sum_{t=1}^{n} e_{t}^{2}=\sum_{t=1}^{n}\left[\sum_{i=1}^{2} K_{i} e_{i t}\right] \\
& =\left[\begin{array}{ll}
K_{1} & K_{2}
\end{array}\right]\left[\begin{array}{ll}
\sum_{t=1}^{n} e_{1 t}^{2} & \sum_{t=1}^{n} e_{1 t} e_{2 t} \\
\sum_{t=1}^{n} e_{2 t} e_{1 t} & \sum_{t=1}^{n} e_{2 t}^{2}
\end{array}\right]\left[\begin{array}{l}
K_{1} \\
K_{2}
\end{array}\right]=K^{T} E K
\end{aligned}
$$

The marginal contribution rate solution model:

$$
\left\{\begin{array}{l}
\min f=\min K^{T} E K \\
\text { s.t } \quad \mathrm{U}^{T} K=1
\end{array}\right.
$$

In order to satisfy $K_{1}+K_{2}=1$, in the formula, the $U^{T}=[1,1]$, then Lagrange multiplier is used to solve the objective function, which:

$$
K=\frac{E^{-1} U}{U^{T} E^{-1} U}
$$

\section{Case Study}

This paper uses the load data of industrial and commercial users in a certain area of Tokyo as a reference for verification (data from Tokyo Electric Power Company). Figure 2 shows the historical load of 24 hours per day for industrial and commercial users in the region from January 1 to 10,2019 (excluding holidays) data.

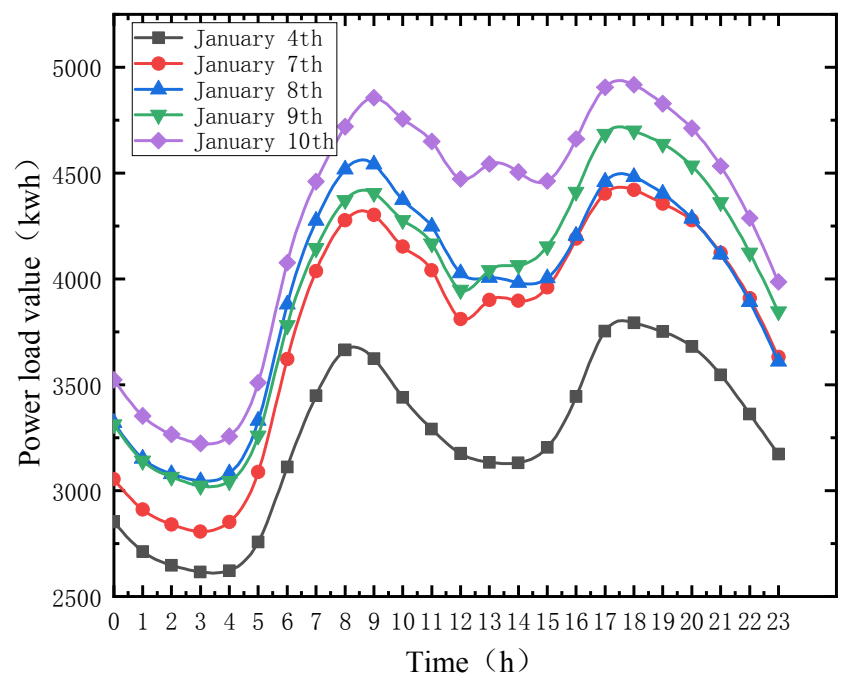

Figure 2. Comparative analysis of historical load data.

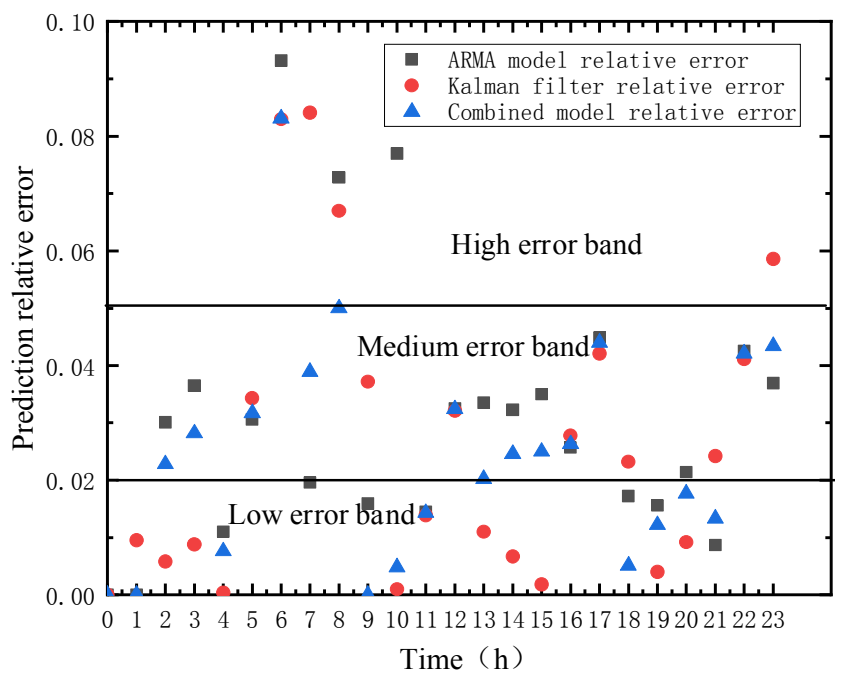

Figure 3. Comparison of prediction curves.

From Figure 2, the load of industrial and commercial users in this area fluctuates greatly over time, and there is obvious peak and valley period. The traditional single prediction model is insufficient for such load characteristics. To verify the prediction accuracy of the combined prediction model, this paper uses MATLABR2016b and EVIEWS respectively obtain the prediction curve and relative error of the Kalman 
filter model and the ARMA model, as shown in Figure 3 and Figure 4. Finally, the combined prediction results are obtained by the Shapley value method, as shown in Table 1.

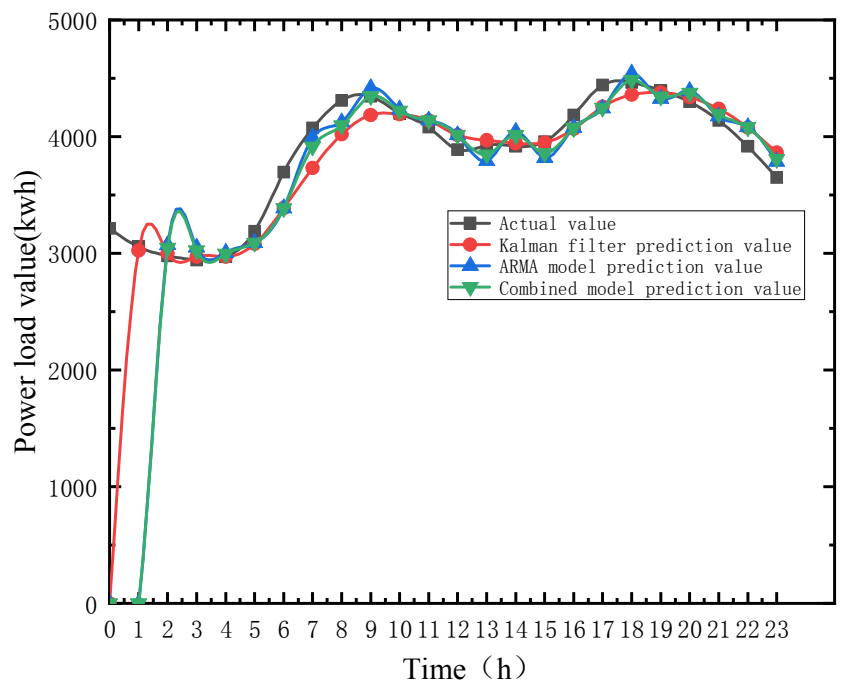

Figure 4. Relative error distribution.

In the combined prediction model, the marginal contribution rate of the Kalman filter is 0.7015 , and the marginal contribution rate of the ARMA model is 0.2985. This is because the average relative error of the Kalman filter prediction model is lower than that of the ARMA model. From the prediction results in Table 1, it can be concluded that the power consumption is relatively low and relatively stable during the period from 1 to 4 in the morning, and the relative error of the Kalman filter model is up to 0.0095, which is much smaller than the 0.0396 of the ARMA model. Similarly, between $10: 00$ to $15: 00$, the relative error of the Kalman filter model is much smaller than that of the ARMA model. Reflected in Figure 3, the relative error of the Kalman filter is in the low error band. During the period of $6-9$, the user's power consumption gradually increases. The fluctuation characteristics are obvious. The highest relative error of the ARMA model is not much different from the Kalman filter model. However, the average relative error of the ARMA model is much smaller than the Kalman filter model. It is reflected in Figure 4. The relative error of the Kalman filter is in the high error band. The average relative error of the ARMA model is less than the Kalman filter model during the period of $15-24$ points. After the combination, the relative error of the combined prediction model is small during the whole prediction process, and the overall relative error is in the middle and low error band. The influence of load fluctuation caused by time factor is large, and the average relative error is smaller than the single prediction model.

Table 1. Evaluation information of expert linguistic variables.

\begin{tabular}{|c|c|c|c|c|c|c|c|}
\hline $\begin{array}{l}\text { Predict point } \\
\text { in time }\end{array}$ & $\begin{array}{l}\text { Measured } \\
\text { values }\end{array}$ & $\begin{array}{l}\text { Kalman Filter } \\
\text { Predictive Value }\end{array}$ & $\begin{array}{l}\text { Relative } \\
\text { error }\end{array}$ & $\begin{array}{l}\text { ARMA Model } \\
\text { Predictive value }\end{array}$ & $\begin{array}{l}\text { Relative } \\
\text { error }\end{array}$ & $\begin{array}{l}\text { Combination Model } \\
\text { Predictive values }\end{array}$ & $\begin{array}{l}\text { Relative } \\
\text { error }\end{array}$ \\
\hline 0 & 3213.6 & - & - & - & - & - & - \\
\hline 1 & 3054.2 & 3025.0 & 0.0095 & - & - & - & - \\
\hline 3 & 2943.4 & 2969.3 & 0.0088 & 3050.9 & 0.0365 & 3026.5 & 0.0282 \\
\hline 4 & 2972 & 2970.7 & 0.0004 & 3004.8 & 0.0110 & 2994.6 & 0.0076 \\
\hline 5 & 3189.6 & 3080.1 & 0.0343 & 3091.9 & 0.0306 & 3088.4 & 0.0317 \\
\hline 7 & 4073.6 & 3730.6 & 0.0841 & 3993.4 & 0.0196 & 3914.9 & 0.0389 \\
\hline 8 & 4310.6 & 4021.5 & 0.0670 & 4126.0 & 0.0428 & 4094.8 & 0.0500 \\
\hline 9 & 4346.4 & 4184.6 & 0.0372 & 4415.6 & 0.0159 & 4346.6 & 0.0000 \\
\hline 10 & 4200.4 & 4192.1 & 0.001 & 4233.1 & 0.0077 & 4220.8 & 0.0048 \\
\hline 11 & 4079.8 & 4136.7 & 0.0139 & 4139.3 & 0.0145 & 4138.5 & 0.0143 \\
\hline 12 & 3887.4 & 4012.2 & 0.0321 & 4014.0 & 0.0325 & 4013.4 & 0.0324 \\
\hline 13 & 3925.4 & 3968.8 & 0.0110 & 3793.6 & 0.0335 & 3845.9 & 0.0202 \\
\hline 14 & 3916.4 & 3942.7 & 0.0067 & 4042.9 & 0.0323 & 4013.0 & 0.0246 \\
\hline 16 & 4183.2 & 4066.4 & 0.0278 & 4075.5 & 0.0257 & 4072.8 & 0.0263 \\
\hline 17 & 4442 & 4254.6 & 0.0421 & 4242.5 & 0.0449 & 4246.1 & 0.0440 \\
\hline 18 & 4463.2 & 4359.2 & 0.0232 & 4540.2 & 0.0172 & 4486.1 & 0.0051 \\
\hline 19 & 4395.2 & 4377.5 & 0.0040 & 4326.2 & 0.0156 & 4341.5 & 0.0122 \\
\hline 20 & 4298.6 & 4338.2 & 0.0092 & 4390.7 & 0.0214 & 4375.1 & 0.0177 \\
\hline 21 & 4137 & 4237.3 & 0.0242 & 4173.1 & 0.0087 & 4192.2 & 0.0133 \\
\hline 22 & 3915.8 & 4076.7 & 0.0411 & 4082.4 & 0.0425 & 4080.7 & 0.0421 \\
\hline 23 & 3649.4 & 3863.4 & 0.0586 & 3784.3 & 0.0369 & 3807.9 & 0.0434 \\
\hline
\end{tabular}

\section{Conclusion}

In the actual type of electricity consumption, the residential electricity demand is relatively stable, and the space of participating in the demand response is small, while the industrial and commercial users generally use a large amount of electricity, and the electricity demand can be adjusted according to the real-time operation of the power grid. The key premise of the main participants, while evaluating the effectiveness of the demand response project implementation is to accurately predict the user's baseline 
load. In this paper, the Kalman filter and the ARMA model are combined with each other to avoid the problem of Kalman filter prediction in the period of stable load fluctuation, and the ARMA model has large prediction bias; in the period of large load fluctuation The Kalman filter predicts a large deviation, and the ARMA predicts more accurately. Therefore, the combined forecasting model reduces the influence of time factor causing large deviation of load forecasting without changing the advantages of the original single forecasting model, making up for the shortcomings of the single model and improving the overall prediction accuracy. The example verifies that the combined model is effective and feasible.

\section{References}

[1] ZHANG Qin, WANG Xifan, WANG Jianxue, et al. Survey of Demand Response Research in Deregulated Electricity Markets [J]. Auto-mation of Electric Power System, 2008, 32 (3): 97-1065.

[2] US Department of Energy. Benefits of demand response in electricity markets and recommendations for achieving them: a report to the United State Congress pursuant to section 1252 of the Energy Policy Act of 2005 [EB/OL]. [2007-07-21]. http://www.oe.energy.gov/Docume-nts and Media/congress_1252d.pdf.

[3] SUN Jianlong, ZHOU Zongrong, TAN Jian, et al. Research on Calculation Method of User Baseline Load in Demand Response [J]. E-lectronic Technology Application, 2014, (z1): 202-205, 208.

[4] NIU Wenjuan, WANG Lei, LI Yang. Calculation method andapplication of customer baseline load in demand response project [J]. JOURNAL OF SOUTHEAST UNIVERSITY (Natural Science Edition), 2014, 44 (3): 556-560.

[5] YU Sheng, ZOU Hongbo, YU Fan. et al. Application of fuzzy neural network in power short-term load forecasting [J]. Smart power. 2018, 46 (11): 88-91+97.
[6] WANG Huizhong, ZHOU Jia, LIU Ke. Summary of Research on the Short-term Load Forecasting Method of the Electric Power System. Electrical Automation. 2015, 37 (01): 1-3+39.

[7] HU Yang, CHANG Xianrong. Short-term load forecasting based on improved EMD-PSVM [J]. Shaanxi Electric Power, 2016, 44 (03): 29-33.

[8] QU Dapeng, WU Weihua, JIANG Di, et al. Calculation method of radial basis function neural network based on user demand side response baseline load [J]. ELECTRONIC TEST, 2014 (2X): 26-30.

[9] LIU Guishun, YANG Jiaju, WANG Lei. Baseline load calculation with considering customer different electrical characterist-ics [J]. POWER DSM, 2016 (3): 17-22.

[10] WANG Xiaofeng, SU Huilin, SONG L T, HUANG Q H. Differential Customer Baseline Load Forecasting Based on Load Subdivision [J]. Electric Power Engineering Technology, 2018, 37 (06): 33-38.

[11] Grimm C, Energy D T E. Evaluating baselines for demand response programs [C]//AEIC Load Research Workshop. 2008.

[12] Coughlin K, Piette M A, Goldman C, et al. Estimating demand response load impacts: Evaluation of baseline load models for non-residential buildings in California [J]. 2008.

[13] Woo C K, Herter K. Residential demand response evaluation: a scoping study $[\mathrm{J}]$. Energy and Environmental Economics, Inc. and Lawrence Berkeley National Laboratory, 2006.

[14] PENG Dingcong. Basic principle and application of Kalman [J]. Software Guide, 2009 (11).

[15] WANG Zhenglong, HU Yonghong. Application time series analysis [J]. Beijing: Science Publis-hing. Society, 2007 (in Chinese)

[16] LI Lingchuan, LV Dong, WU Wenjie. A linear combination based simplified load forecasting method for power system [J]. Power System Technology, 2002 (10): 10-13. 